\title{
Labyrinthe
}

22 | 2005 (3)

La Biopolitique (d')après Michel Foucault

\section{Population, milieu et normes}

Note sur l'enracinement biologique de la biopolitique de Foucault

\section{Charles Ruelle}

\section{(2) OpenEdition}

Journals

Édition électronique

URL : http://journals.openedition.org/labyrinthe/1031

DOI : $10.4000 /$ labyrinthe. 1031

ISSN : 1950-6031

Éditeur

Hermann

Édition imprimée

Date de publication : 1 novembre 2005

Pagination : 27-34

\section{Référence électronique}

Charles Ruelle, «Population, milieu et normes », Labyrinthe [En ligne], 22 | 2005 (3), mis en ligne le 21

juillet 2008, consulté le 20 avril 2019. URL : http://journals.openedition.org/labyrinthe/1031 ; DOI :

10.4000/labyrinthe. 1031

Propriété intellectuelle 


\title{
POPULATION, MILIEU ET NORMES Note sur l'enracinement biologique de la biopolitique de Foucault
}

\author{
Charles Ruelle \\ charles.ruelle@freesbee.fr
}

Dans La volonté de savoir, faisant référence au droit de vie et de mort du souverain sur ses sujets, Foucault analyse les formes anciennes et classiques du pouvoir comme ce que l'on pourrait appeler un thanatopouvoir. Dans ce cadre, le souverain n'exerce son droit sur la vie « qu'en faisant jouer son droit de tuer, ou en le retenant; il ne marque son pouvoir sur la vie que par la mort qu'il est en mesure d'exiger »(VS, p. 178). Dans ce cas, le pouvoir sur la vie n'est qu'un pouvoir sur la mort, un pouvoir indirect qui s'exerce dans la négation de la vie. À l'inverse, le biopouvoir s'exerce positivement sur la vie, afin de mieux la gérer, « de la majorer, de la multiplier, d'exercer sur elle des contrôles précis et des régulations d'ensemble » (VS, p. 180). Il s'agit d'investir le pouvoir au cœur de la vie, de mieux la contrôler pour faire émerger encore plus de vie. À l'extériorité, par rapport à la vie, des formes anciennes du pouvoir s'oppose l'intériorité du biopouvoir sur la vie. À la négation de la vie s'oppose une action positive au cœur même de cette vie. Mais, de fait, le pouvoir présuppose toujours dans les deux cas la vie comme un donné sur lequel il agit ou n'agit pas. C'est pourquoi la distinction entre les anciennes formes du pouvoir et le biopouvoir ne peut être ainsi résumée dans ces oppositions trop fluides.

En effet, le biopouvoir semble n'être ici qu'une variété particulière d'une même espèce du pouvoir qui, d'une manière générale, a toujours besoin de la vie pour exister. Dans ce cadre, l'analyse du biopouvoir exige seulement de savoir ce qu'est un pouvoir qui investit la vie par rapport à un pouvoir indirect sur la vie. Elle exige que l'on s'interroge uniquement sur les modalités d'action et les dispositifs du pouvoir lui-même. La vie, en revanche, ne semble pas poser de problème. Si pour le sens commun, 
toutes les formes politiques du pouvoir - anciennes, modernes (libérales) ou contemporaines (néo-libérales) - apparaissent toujours comme un pouvoir de la vie sur la vie, de l'homme sur l'homme, une telle conception n'épuise pas le concept et la nature du biopouvoir, et contredit le constructivisme foucaldien sur la nature même de la vie. Pour Foucault, en effet, la vie est une catégorie humaine plus que naturelle. Son existence n'est pas donnée une fois pour toutes dans la réalité, mais dépend des structures conceptuelles que l'homme met en place pour l'appréhender. Sur ce point, la thèse de Foucault est célèbre: « on veut faire des histoires de la biologie au XVIII ${ }^{\mathrm{e}}$ siècle; mais on ne se rend pas compte que la biologie n'existait pas [...]. Et que si la biologie était inconnue, il y avait à cela une raison bien simple: c'est que la vie elle-même n'existait pas $^{1}$.»

Il est clair, dès lors, que l'émergence d'un biopouvoir, d'un pouvoir sur la vie, ne peut se produire que dans le contexte contemporain, ou postérieur à, l'invention de la vie elle-même $\rightarrow$ voir l'article d'A. Skornicki $\nleftarrow$. Or, de ce point de vue, l'apparition du mot biologie à la fin du XVIII ${ }^{\mathrm{e}}$ siècle, offre un point d'ancrage tout à fait significatif ${ }^{2}$. De plus, l'émergence du biopouvoir ne peut être ici évaluée à la mesure d'un simple changement de perspective, d'un déplacement de l'exercice externe du pouvoir sur la vie à un investissement interne. On manquerait alors l'originalité fondamentale du biopouvoir, à savoir qu'il n'est pas une forme nouvelle du gouvernement des vivants. Il est plutôt la seule modalité jamais éprouvée du pouvoir sur la vie. Autrement dit: l'émergence du biopouvoir n'est pas seulement la mise en place de nouveaux dispositifs de contrôles sur la vie, mais la réponse politique à l'émergence d' un nouvel objet du pouvoir, à savoir la vie elle-même. Reste alors à se demander, pour échapper à la tautologie, quelle est cette vie qui est au cœur du biopouvoir? À quoi fait référence ce bios dans lequel s'ancre la biopolitique? C'est à travers une revue des principaux concepts biologiques dans lesquels s'instancie le vitalisme de la biopolitique que nous envisageons notre analyse et entendons fournir des pistes pour une exégèse future plus approfondie.

1. Les Mots et les choses, Paris, Gallimard, coll. « Tel», 1990, p. 139.

2. Voir Peter McLaughlin, « Naming Biology », Journal of the History of Biology, vol. 35, $\mathrm{n}^{\circ}$ 1, 2002, p. $1-4$. 


\section{De la biologie à la biopolitique}

Il apparaît assez clairement que Foucault donne au bios une détermination proprement biologique et que cette dimension fondamentale et première du sujet/objet du pouvoir règle les fins de la conduite politique. Dans La volonté de savoir, décrivant l'opposition entre souveraineté et biopouvoir, Foucault écrit ainsi que « le pouvoir d'exposer une population à une mort générale est l'envers du pouvoir de garantir à une autre son maintien dans l'existence. Le principe: pouvoir tuer pour pouvoir vivre, qui soutenait la tactique des combats, est devenu principe stratégique entre États; mais l'existence en question n'est plus celle, juridique, de la souveraineté, c'est celle, biologique, d'une population » (VS, p. 181). De même, dans Sécurité, territoire, population, Foucault définit le biopouvoir comme «l'ensemble des mécanismes par lesquels ce qui, dans l'espèce humaine, constitue ses traits biologiques fondamentaux va pouvoir entrer à l'intérieur d'une politique, d'une stratégie politique, d'une stratégie générale de pouvoir, autrement dit comment la société, les sociétés occidentales modernes, à partir du XVIII ${ }^{\mathrm{e}}$ siècle, ont repris en compte le fait biologique que l'être humain constitue une espèce humaine » (STP, p. 3$)$.

Partant d'une telle définition, on pourrait s'attendre à ce que Foucault tente une entreprise de type sociobiologique, c'est-à-dire une justification du libéralisme biopolitique à partir d'une analyse anthropologique de la nature humaine. Ce n'est pas le cas. Si la rationalité politique se superpose aux transformations de la rationalité biologique, elle n'entretient pas avec elle un rapport de nécessité logique, mais adaptative. Foucault ne fonde pas son analyse à partir d'une clarification des traits biologiques de l'espèce. Au contraire, la définition du «biopouvoir» donnée ci-dessus ouvre directement sur l'analyse des mécanismes et des dispositifs que met en œuvre le biopouvoir (STP, p. 3), puis des traits généraux des dispositifs de sécurité (STP, p. 13). De ces dispositifs émergent toutefois d'autres indices de la nature biologique de l'objet analysé.

Foucault distingue quatre traits généraux des dispositifs de sécurité: le milieu comme espace de sécurité, le traitement de l'aléatoire par régulations, l'inscription de la norme à l'intérieur de normalités différentielles, la population comme sujet et objet des mécanismes sécuritaires. Ici, et contrairement au système disciplinaire, l'analyse des dis- 
positifs sécuritaires emprunte ses principaux concepts, ou leur assigne un sens commun, à l'épistémologie biologique et médicale de la seconde moitié du XIX ${ }^{\mathrm{e}}$ siècle. L'opposition entre le disciplinaire et le sécuritaire se joue ainsi dans l'articulation entre les anciens concepts prébiologiques et les autres: entre le territoire et le milieu, la normation et la normalisation, le peuple et la population, l'instruction et la régulation. Il est alors possible d'interpréter la biopolitique comme un témoignage de la dynamique qu'a opérée, dans le champ de l'action politique, la substitution de concepts «darwiniens » à des concepts contemporains de l'histoire naturelle. Elle révèle, en outre, au cœur des dispositifs sécuritaires la prise en compte de cette nouvelle dimension proprement biologique de l'objet politique.

Dans ce cadre, le concept de population est la pierre angulaire de tout l'édifice sur lequel se fonde notamment la biopolitique. Il est, selon Foucault, « l'opérateur de transformation qui a fait passer de l'histoire naturelle à la biologie, [mais aussi] de l'analyse des richesses à l'économie politique, de la grammaire générale à la philologie historique» (STP, p. 80). La biopolitique, autant qu'une « politique de la vie » est une politique de la population biologique. Il convient dès lors de se demander dans quelle mesure la conception foucaldienne de la population est-elle révélatrice de l'ancrage biologique de la biopolitique?

\section{La biopolitique comme politique de la population}

Si le terme de population apparaît en anglais dès 1751 sous la plume de David Hume dans ses Political Discourses ${ }^{3}$, il n'en est pas moins vrai, rappelle Foucault, que Darwin « a été le premier à traiter des êtres vivants au niveau de la population, et non plus au niveau de l'individualité» (DE, IV, p. 160). Pour l'ensemble des biologistes depuis Darwin, une population est, dans son sens général, un ensemble d'individus appartenant à la même espèce. Pour Foucault, «la population, c'est [...] tout ce qui va s'étendre depuis l'enracinement biologique par l'espèce jusqu'à la surface de prise offerte par le public» (STP, p. 77). Or, par public, il faut entendre « la population prise du côté de ses

3. Voir l'avant-propos au livre dirigé par Hervé Le Bras, L'Invention des populations, Paris, Odile Jacob, 2000 . 
opinions, de ses manières de faire, de ses comportements, de ses habitudes, de ses craintes de ses préjugés, de ses exigences, c'est ce sur quoi on a prise par l'éducation, par les campagnes, par les convictions » (STP, p. 77). La population est ainsi tiraillée entre une détermination biologique fondamentale du vivant (l'espèce) et une dimension émergente psycho-politique (le public), entre lesquelles elle tisse un lien continu, déterminant ainsi « l'espace pertinent à l'intérieur duquel et à propos duquel on [i.e. le gouvernement] doit agir» (STP, p. 77). Bien que le sens de « population » soit toujours tiraillé entre diverses déterminations - biologique, démographique, statistique ou historique $\Rightarrow$ voir l'article de D. Cohen $\longleftarrow-$, le niveau biologique offre donc pour Foucault le point d'ancrage fondamental de signification.

Ainsi, quand Foucault détermine la spécificité de la population, c'est en révélant son caractère quasi organique: aux « éléments » du peuple qui entretiennent entre eux, et par rapport au peuple, une relation anorganique d'extériorité, et d'indépendance, Foucault oppose les «membres » de la population considérée comme un espace de régulations et d'échange avec le milieu.

Il est vrai, jamais Foucault ne cède à la tentation d'une qualification de la population en terme d'organisme ou de totalité, semblant vouloir échapper à une analyse trop vitaliste. Cependant, son interprétation des signes de la «naturalité » (STP, p. 72) de la population pourrait de fait témoigner en faveur de sa vitalité, notamment: sa dépendance vis-à-vis des variables qu'offrent le milieu (les lois qui la régissent, les habitudes de ses membres, ses valeurs, ses ressources), la constance de phénomènes qui dépendent du hasard et de causes conjoncturelles irréductibles à un mécanisme de type cartésien, ou « la production de l'intérêt collectif par le jeu du désir».

D'où cette autre définition foucaldienne de la population comme « ensemble d'éléments à l'intérieur duquel on peut repérer l'universel du désir produisant régulièrement le bénéfice de tous, et à propos duquel on peut repérer un certain nombre de variables dont il est dépendant et qui sont susceptibles de le modifier » (STP, p. 76). Cette variabilité confère à la population une autonomie par rapport à la volonté unique du souverain. Or, c'est relativement à cette autonomie de la population par rapport à toute action extérieure directe du souverain que le milieu apparaît comme l'espace privilégié de la biopolitique. Qu'est-ce que le milieu où se joue la biopolitique? 


\section{Le milieu comme nouvel espace de la biopolitique}

Foucault envisage ici son analyse (STP, p. 28-29) en s'appuyant sur l'article de Canguilhem, "Le vivant et son milieu ${ }^{4} »$. Canguilhem a montré que le terme de milieu a reçu, à l'origine, une signification mécanique. D'abord présent chez Newton, il était conçu comme « fluide véhicule d'action à distance ${ }^{5}$ » entre deux corps. Foucault reprend à son compte cette idée, définissant le milieu comme « ce qui est nécessaire pour rendre compte de l'action nécessaire d'un corps sur un autre. C'est donc bien le support et l'élément de circulation d'une action» (STP, p. 22). Le terme de milieu est introduit en biologie par Lamarck. À ce propos, l'éditeur du cours, Michel Senellart, cite en note un extrait de l'article de Canguilhem: «Lamarck parle toujours de milieux, au pluriel, et entend par là expressément des fluides comme l'eau, l'air et la lumière. Lorsque Lamarck veut désigner l'ensemble des actions qui s'exercent du dehors sur un vivant [...], il ne dit jamais le milieu, mais toujours "circonstances influentes". Par conséquent, circonstances est pour Lamarck un genre dont climat, lieu et milieu sont les espèces. » (STP, p. 29.) Or, c'est en traçant un parallèle quasi parfait avec la conception lamarckienne du milieu que Foucault analyse la gestion sécuritaire des espaces, en insistant sur la circulation des fluides. « La discipline travaille dans un espace vide, artificiel, que l'on va construire entièrement. La sécurité, elle, va prendre appui sur un certain nombre de données matérielles. Elle va travailler bien sûr l'emplacement avec l'écoulement des eaux, avec les îles, avec l'air, etc. Donc, elle travaille sur un donné. » (STP, p. 21.) Dans la politique sécuritaire, il s'agit alors d'aménager un milieu pour une population, en fonction d'un donné et de séries d'événements aléatoires.

L'action biopolitique, en ce sens, apparaît fondamentalement comme une action indirecte sur la population par le biais des transformations et de l'aménagement du milieu. La conséquence inévitable en est sans doute que la biopolitique ne peut jamais véritablement s'éloigner de toute tentation eugénique, déjà présente chez Jean-Baptiste Moheau, que Foucault considère comme «le premier grand théoricien» de la biopolitique, et

4. Article cité dans La Connaissance de la vie, Paris, Vrin, 1989, p. 129-154.

5. Ibidem, p. 130. 
dont l'ambition n'était ni plus ni moins de changer l'espèce humaine (STP, p. 24). Là, il ne s'agit plus seulement de rendre les gens, les gestes, les actes, conformes à un modèle, à une norme, comme dans le modèle disciplinaire. Il s'agit d'adapter le comportement du plus grand nombre - dans des limites statistiques considérées comme acceptables - à de nouvelles normes. Quel traitement la biopolitique fait-elle de la norme sociale? Quelles procédures utilise-t-elle pour imposer un système de normes à la population?

\section{De la normation disciplinaire à la normalisation sécuritaire}

Dans le cadre de la biopolitique, il s'agit de faire en sorte que la norme émerge du comportement global de la population. Le contrôle des comportements passe alors, dans le cadre de la biopolitique, à travers une inoculation, au sens propre et figuré, de la norme sociale à travers la mise en place de procédures relevant d'une médecine sociale susceptible d'imposer et de modifier de nouvelles normes de vie, « qu'il s'agisse de l'alimentation, de l'habitat ou de l'aménagement des villes» (STP, p. 377).

Le traitement de la norme sociale se révèle ainsi très différent selon que l'on se trouve dans le contexte disciplinaire ou sécuritaire. Dans le cadre disciplinaire, le normal est ce qui est capable de se conformer à une norme posée comme moyen de certaines fins, l'anormal, ce qui n'en est pas capable. Ici, c'est toujours la norme, la loi, qui est première. Ce n'est pas le normal et l'anormal. «Autrement dit, il y a un caractère primitivement prescriptif de la norme, et c'est par rapport à cette norme posée que la détermination et le repérage du normal et de l'anormal deviennent possibles. » (STP, p. 59.) Dans le contexte sécuritaire, la «norme est un jeu à l'intérieur des normalités différentielles » (STP, p. 65). Ici, le traitement de la norme que propose Foucault doit une nouvelle fois beaucoup aux analyses de Canguilhem, dans la première partie de son livre Le Normal et le pathologique. Les processus de normalisation de la biopolitique répondent en effet à une thèse largement répandue dans la médecine positive du XIX ${ }^{e}$ siècle, à savoir celle de la continuité du normal et de l'anormal, de l'identité du normal et du pathologique, selon laquelle « les phénomènes pathologiques ne sont dans les organismes vivants rien de plus que des variations quantitatives, selon le plus et le moins, des 
phénomènes physiologiques correspondants ${ }^{6}$. » Dans le cadre du traitement de la variole analysé par Foucault - exemplaire d'un dispositif sécuritaire au XVIII ${ }^{\text {e }}$ siècle -, la rupture entre le normal et l'anormal disparaît elle aussi. Le dispositif de traitement ne fait aucun partage. Il consiste « à prendre en considération l'ensemble sans discontinuité, sans rupture, des malades et non malades, c'est-à-dire en somme la population, et à voir dans cette population quel est le coefficient de morbidité probable» $(S T P$, p. 64). On n'agit plus sur les individus mais sur les groupes à risque $\rightarrow$ voir l'article de D. Moreau $\leftrightarrow$. On n'a plus le sain et le non-sain, le normal et l'anormal, on a divers niveaux de courbes d'où l'on déduit des populations, aujourd'hui dites «à risque ». La technique va alors consister à ramener à la normalité fixée par des courbes statistiques les groupes les plus défavorables, les plus déviants par rapport à la courbe normale, générale. Ce qui est normal, ce n'est plus ce qui est prescrit, ce sont les diverses distributions. C'est pourquoi, selon Foucault, la normalisation sécuritaire correspond plus à une normalisation qu'à une véritable «normation » (STP, p. 59).

De là à dire que la construction des normes de la biopolitique est issue du champ conceptuel de la médecine positive, il y a un pas qu'il ne faut pas franchir. Elles partagent en revanche une même conceptualité due à la nature commune de leur objet, la population, le vivant et sa capacité à tolérer et éliminer des écarts quantitatifs vis-à-vis d'une norme. De ce point de vue, l'exemple de la variolisation pour illustrer le traitement de la norme sécuritaire est aussi éclairant que trompeur, puisqu'il traite d'un problème propre à la population en général (sa gestion statistique des normes) selon l'angle spécifique de la mise en place d'une politique sanitaire (variolisation). Or, la production de normes sécuritaires, bien qu'inspirée ici de l'analyse des normes biomédicales chez Canguilhem, n'en est pas moins propre à toute une variété de domaines d'intervention (éducation, économie, logement, etc.). Mais il est vrai que l'on peut ici pousser plus loin la métaphore médicale: si la biopolitique, comme nous l'avons suggéré, est en propre la politique de la vie, de la population, l'ensemble de ses procédures ne devrait, en fin de compte et d'une manière générale, avoir d'autre but que celui de lui assurer la santé.

6. Georges Canguilhem, Le Normal et le pathologique, Paris, Puf, 1966, p. 14. 